\title{
Mudanças no padrão de utilização de serviços de saúde no Brasil entre 2013 e 2019
}

\author{
Changes in the pattern of health services use in Brazil between 2013 \\ and 2019
}

\author{
Célia Landmann Szwarcwald (http://orcid.org/0000-0002-7798-2095) ${ }^{1}$ \\ Sheila Rizzato Stopa (https://orcid.org/0000-0001-8847-665X) ${ }^{2}$ \\ Giseli Nogueira Damacena (https://orcid.org/0000-0002-7059-3353) ${ }^{1}$ \\ Wanessa da Silva de Almeida (https://orcid.org/0000-0002-5164-8603) ${ }^{1}$ \\ Paulo Roberto Borges de Souza Júnior (https://orcid.org/0000-0002-8142-4790) ${ }^{1}$ \\ Maria Lúcia França Pontes Vieira (https://orcid.org/0000-0002-5284-6214) ${ }^{3}$ \\ Cimar Azeredo Pereira (https://orcid.org/0000-0001-6183-1607) ${ }^{3}$ \\ Luciana Monteiro Vasconcelos Sardinha (https://orcid.org/0000-0002-3679-3618) ${ }^{2}$ \\ Eduardo Marques Macário (https://orcid.org/0000-0002-6383-0365) ${ }^{2}$
}

${ }^{1}$ Instituto de Comunicação

e Informação Científica

e Tecnológica em Saúde,

Fiocruz. Av. Brasil 4365,

Manguinhos. 21040-360

Rio de Janeiro RJ Brasil.

celia_ls@hotmail.com

${ }^{2}$ Departamento de

Análise em Saúde e

Vigilância de Doenças Não

Transmissíveis, Secretaria

de Vigilância em Saúde,

Ministério da Saúde. Brasília

DF Brasil

${ }^{3}$ Diretoria de Pesquisas,

Instituto Brasileiro de

Geografia e Estatística. Rio

de Janeiro RJ Brasil.

\begin{abstract}
This study aimed to investigate changes in the health service use pattern based on information from the 2013 and 2019 National Health Surveys (PNS). The two outcomes, "Seeking health-related care in the past two weeks" and "Medical visit in the last twelve months", were analyzed according to socioeconomic, geographic and health conditions characteristics. Multivariate Poisson regression models were used to investigate the factors associated with seeking care due to a health problem or prevention. The prevalence of chronic diseases increased from $15.0 \%$ to $22.5 \%$ between 2013 and 2019. The proportion of seeking care increased from 15.3 to $18.6 \%$, and medical visits from $71.2 \%$ to $76.2 \%$, ranging from 61.4 to $75.8 \%$ and 68.0 to $80.6 \%$ between the North and Southeast regions. There was no significant association of seeking care due to a health problem with per capita income, after controlling for the other covariates. We conclude by saying that, despite the expanded coverage of health service use, the persistent regional inequalities indicate unmet health needs among residents of the less developed regions. Health care models focused on prevention and health promotion are required.
\end{abstract}

Key words Health service use, Surveys, Health needs, Inequalities, Brazil
Resumo Este estudo teve o objetivo de comparar os padrões de utilização de serviços de saúde, a partir das informações das edições da Pesquisa Nacional de Saúde (PNS), 2013 e 2019. Os dois desfechos "Procura de atendimento relacionado à saúde nas últimas duas semanas" e "Consulta médica nos últimos doze meses" foram analisados segundo fatores socioeconômicos, geográficos, e condições de saúde. Foram usados modelos multivariados de regressão de Poisson para investigar os fatores associados à procura de atendimento de acordo com o motivo (problema de saúde ou prevenção). Entre 2013 e 2019, a prevalência de doenças crônicas aumentou de $15,0 \%$ a 22,5\%. A proporção de busca de atendimento cresceu de 15,3 a 18,6\%, e de uso de médico, de 71,2\% a $76,2 \%$, com amplitudes de variação de 61,4$75,8 \%$ e 68,0-80,6\% entre as regiões Norte e Sudeste. Para atendimento por problema de saúde, não houve associação significativa com rendimento per capita, após o controle das demais covariáveis. Conclui-se que apesar da expansão da cobertura de utilização de serviços de saúde, as persistentes desigualdades regionais indicam necessidades de saúde não atendidas entre os residentes das regiões menos desenvolvidas. Modelos de atenção focados na prevenção e promoção da saúde são necessários.

Palavras-chave Uso de serviços de saúde, Inquéritos, Necessidades de saúde, Desigualdades, Brasil 


\section{Introdução}

No Brasil, a organização do sistema nacional de saúde é fundamentada em dispor de um sistema público, responsável por prover assistência gratuita e universal a qualquer brasileiro que necessite, e da saúde suplementar, definida por todo atendimento privado de saúde, realizado ou não por meio de um convênio com um plano de saúde.

A criação do SUS pela Constituição de 1988 representou uma importante mudança no padrão de organização dos serviços de saúde no País, focado no fortalecimento da atenção básica de saúde e na descentralização dos serviços, com vistas a aumentar a acessibilidade ao sistema de saúde e incrementar as ações de prevenção e promoção de saúde 1 . Com a implantação do Programa de Saúde da Família (PSF) na década de 1990, foi estabelecido um direcionamento geográfico da atenção por meio de equipes de saúde da família nas áreas e nos subgrupos populacionais desfavorecidos socialmente ${ }^{2}$.

Em meados dos anos 2000, a atenção primária em saúde, considerada a principal porta de entrada ao sistema de saúde, passou a coordenar os cuidados na rede de serviços e a efetivar a integração da rede de serviços ${ }^{3}$. As atuais diretrizes da integração dos cuidados envolvem a existência de um serviço de procura regular, a oferta de serviços de atenção primária e promoção da saúde e a garantia de acesso aos diversos níveis de atenção por meio de mecanismos de referência para assegurar a continuidade da atenção à saúde ${ }^{4}$.

Como parte do processo de descentralização, foram criadas regiões de saúde e estratégias para a integração dos diferentes níveis de atenção. Os municípios desenvolveram propostas buscando viabilizar e otimizar a implantação dos novos modelos de atenção, com critérios de organização definidos pelo governo federal, e outros próprios de gestão municipal, impondo a necessidade de avaliar os processos de implantação e execução das intervenções e os seus resultados sobre a saúde da população ${ }^{5}$.

Pesquisas direcionadas à avaliação do desempenho de saúde sob a perspectiva da população usuária têm tido importância crescente e sido aplicadas em vários países do mundo ${ }^{6-8}$. A percepção do usuário tem sido cada vez mais valorizada, constituindo etapa essencial para o planejamento das ações voltadas para garantir a qualidade da atenção à saúde e subsidiar decisões que atendam às necessidades da população9. No Brasil, a geração periódica de informações de base populacional tem se constituído em uma ferramenta indispensável para monitorar o acesso e a utilização dos serviços de saúde, bem como das condições de saúde da população, permitindo avaliar as políticas públicas de saúde e fornecer subsídios para o seu aprimoramento ${ }^{10-12}$.

Nesse sentido, a busca por atendimento relacionado à saúde e a utilização de consulta médica representam indicadores positivos de acesso ao sistema de saúde à medida que podem ter impacto importante sobre a saúde da população, prevenindo a ocorrência de doenças, reduzindo a morbimortalidade e aumentando a esperança de vida saudável ${ }^{13}$. Adicionalmente, estudos sobre a utilização de serviços de saúde podem contribuir para a organização da assistência, permitindo estabelecer as desigualdades nos níveis de cobertura segundo as características sociodemográficas e o local de residência e identificar os grupos populacionais mais vulneráveis ${ }^{14}$.

O objetivo deste estudo foi o de investigar o perfil de utilização de serviços de saúde em 2019 e analisar sua evolução em relação a 2013, no Brasil, por meio dos dados da Pesquisa Nacional de Saúde (PNS). Na análise, são considerados fatores demográficos e socioeconômicos e indicadores da necessidade de atenção. Utilizando dados das duas edições da PNS, são comparadas as desigualdades regionais e sociais na utilização de serviços de saúde.

\section{Métodos}

A Pesquisa Nacional de Saúde é um inquérito de base domiciliar e âmbito nacional, realizada pelo Ministério da Saúde em parceria com o Instituto Brasileiro de Geografia e Estatística (IBGE), nos anos de 2013 e 2019. A população pesquisada corresponde aos moradores de domicílios particulares permanentes do Brasil, exceto os localizados nos setores censitários especiais (quartéis, bases militares, alojamentos, acampamentos, embarcações, penitenciárias, colônias penais, presídios, cadeias, asilos, orfanatos, conventos e hospitais) e os localizados em terras indígenas.

A amostra da PNS constitui uma subamostra da Amostra Mestra do Sistema Integrado de Pesquisas Domiciliares (SIPD) do IBGE, que consiste em um conjunto de unidades de áreas selecionadas para atender às diversas pesquisas do SIPD obedecendo a uma estratificação por 4 critérios: administrativo, de acordo com a divisão administrativa do Brasil (Unidades da Federação, capitais, regiões metropolitanas); geográfico (distritos e subdistritos de municípios de grande porte); situ- 
ação urbano/rural das unidades de áreas; estatístico, com a finalidade de obter maior precisão das estimativas $^{15}$.

O plano amostral da PNS foi por conglomerado em três estágios de seleção, com estratificação das unidades primárias de amostragem (UPA). No primeiro estágio, a seleção das unidades primárias de amostragem (setores censitários ou composição de setores) foi realizada por amostragem aleatória simples, mantendo a mesma estratificação da Amostra Mestra. No segundo estágio, um número fixo de domicílios particulares permanentes foi selecionado aleatoriamente em cada UPA selecionada no primeiro estágio, a partir do Cadastro Nacional de Endereços para Fins Estatísticos (CNEFE). No terceiro estágio, dentro de cada domicílio na amostra, um morador (com 18 ou mais anos de idade, em 2013, e com 15 anos ou mais de idade, em 2019) foi selecionado com equiprobabilidade, a partir de uma lista de moradores elegíveis construída no momento da entrevista, para responder à entrevista individual ${ }^{15}$.

A PNS teve aprovação da Comissão Nacional de Ética em Pesquisa (CONEP) em julho 2013 para a edição de 2013, e em agosto de 2019 para a edição de 2019.

O questionário da PNS é subdividido em três partes, o domiciliar, o de todos os moradores do domicílio e o individual. Os questionários domiciliar e de todos os moradores do domicílio são respondidos por um residente do domicílio que saiba informar sobre a situação socioeconômica e de saúde de todos os moradores. O questionário individual é respondido por um morador selecionado com equiprobabilidade entre todos os residentes adultos do domicílio. Na PNS-2013 foram incluídos moradores com 18 anos ou mais para responder à entrevista individual enquanto na PNS-2019, moradores com 15 anos ou mais.

O trabalho de campo das duas edições da PNS foi de responsabilidade do IBGE. Em 2013, ao final do trabalho de campo, 69.994 domicílios estavam ocupados, e foram realizadas 64.348 entrevistas domiciliares. Em 2019, foram visitados 108.525 domicílios e foram realizadas 94.114 entrevistas. As taxas de não resposta foram, respectivamente, de $8,1 \% \mathrm{e}$ $6,4 \%$.

Os fatores de expansão foram calculados pelo inverso do produto das probabilidades de seleção em cada estágio, incluindo um fator de correção para as perdas. Após a ponderação das bases pelos fatores naturais de expansão, foi realizado um processo de calibração com base nas projeções populacionais para o Brasil e para as Unidades da Federação. Para permitir compara- ções entre as duas edições da PNS, o IBGE recalibrou os fatores de expansão da PNS $2013^{16}$.

A construção do questionário da PNS 2019 seguiu a lógica de prover uma comparação com os dados da PNS 2013 com vistas a possibilitar o monitoramento de indicadores de saúde que são acompanhados espaço-temporalmente ${ }^{17}$. No presente estudo, foram analisadas e comparadas as informações do questionário de todos os moradores do domicílio relativas à procura e à utilização de serviços de saúde.

Foram considerados dois desfechos. O primeiro referiu-se à "Procura de atendimento relacionado à saúde nas últimas duas semanas anteriores à pesquisa", com base na seguinte questão: Nas duas últimas semanas, ___ procurou algum lugar, serviço ou profissional de saúde para atendimento relacionado à própria saúde? Adicionalmente, foram considerados os motivos de procura, e o indicador foi desagregado em dois outros: "Procura de atendimento por motivo de doença ou problema de saúde" e "Procura de atendimento para prevenção". O segundo referiu-se à "Consulta médica nos últimos doze meses anteriores à pesquisa”, que foi baseado na seguinte questão: "Quando foi a última vez que o(a) $\mathrm{Sr}$ (a) consultou um médico?", e foram considerados os indivíduos que tiveram pelo menos uma consulta médica nos últimos 12 meses anteriores à pesquisa.

Os desfechos foram analisados segundo indicadores demográficos (sexo, raça/cor da pele, faixa de idade), regiões de residência, e situação urbana/ rural do setor de residência. A análise incluiu ainda classes de rendimento per capita em termos de salário-mínimo (SM) e posse de plano de saúde.

Foram considerados, igualmente, indicadores de necessidade de atendimento à saúde, estabelecidos pelas seguintes variáveis: avaliação do estado de saúde (bom, regular, ruim); ter diagnóstico de uma doença crônica, física ou mental, ou doença longa de mais de 6 meses de duração (não, sim sem limitações das atividades habituais, sim com limitações das atividades habituais); e problema de saúde nas duas últimas semanas, com base na pergunta "Nas duas últimas semanas, quantos dias ___ deixou de realizar suas atividades habituais, por motivo da própria saúde?”.

Adicionalmente, na análise, considerou-se o indicador "Ter uma fonte usual de cuidados" com base na seguinte questão: “__ costuma procurar o mesmo lugar, mesmo médico ou mesmo serviço de saúde quando precisa de atendimento de saúde?" No caso afirmativo, os tipos de fonte usual de cuidados foram categorizados em: unidade básica de saúde (UBS); unidade de pronto-atendimento 
(UPA); outro serviço público; consultório particular; pronto-atendimento privado; outro.

A análise estatística consistiu em comparar as distribuições dos indivíduos pesquisados em 2013 e 2019 segundo os fatores sociodemográficos, geográficos, e das condições de saúde. A comparação foi realizada pelas estimativas das proporções e respectivos intervalos de confiança de $95 \%$ em cada categoria das variáveis consideradas no estudo.

Para verificar as possíveis mudanças nos indicadores de utilização de serviços de saúde entre 2013 e 2019, os desfechos "Procura de atendimento relacionado à saúde nas duas últimas semanas" e "Uso de consulta médica no último ano" foram analisados segundo os fatores sociodemográficos, geográficos, e de condições de saúde, estimando-se as prevalências e os respectivos intervalos de confiança de $95 \%$ por todas as categorias de cada variável do estudo. Para comparação das prevalências, foi utilizado o teste $t$ de Student de amostras independentes considerando-se diferenças significativas no nível de $1 \%$. Para valores de p (nível descritivo de significância do teste) próximos a $1 \%$, as diferenças foram consideradas limítrofes.

Considerando como variáveis-resposta "Procura de atendimento por motivo de doença ou problema de saúde" e "Procura de atendimento para prevenção", e "Consulta médica nos últimos doze meses anteriores à pesquisa" foram utilizados modelos multivariados de regressão de Poisson para identificar os fatores mais associados aos desfechos, com os dados da PNS de 2013 e 2019, entre os indivíduos com 30 anos ou mais de idade. Primeiramente, foram usadas como covariáveis: idade, sexo, regiões de residência, situação urbana/rural, ter uma fonte usual de cuidados, e rendimento per capita. Para controlar a variância do rendimento per capita, foi utilizada a transformação logarítmica. Posteriormente, substituiu-se a variável de rendimento per capita por posse de plano. Devido à colinearidade entre rendimento per capita e posse de plano, as duas covariáveis foram utilizadas separadamente nos modelos de regressão de Poisson. Foram estimadas as razões de prevalências e foram feitos testes de significância no nível de $1 \%$.

$\mathrm{Na}$ análise estatística de dados, considerouse o desenho de amostragem das duas edições da PNS, levando em conta as ponderações amostrais e o efeito de conglomeração. Utilizou-se o Software for Statistics and Data Science (StataCorp LP, CollegeStation, Texas, United States), versão 14.0, módulo "survey".

\section{Resultados}

Em 2013, 205.546 pessoas participaram da PNS, $48,1 \%$ do sexo masculino e $51,9 \%$ do feminino. Em 2019, participaram 279.382 indivíduos, 47,8\% homens e 52,2\% mulheres. Comparando-se as distribuições por faixa de idade em 2013 e 2019, percebe-se o envelhecimento da população brasileira: no período de 6 anos, o percentual de idosos aumentou de $13,2 \%$ a $16,4 \%$. No tocante à raça/cor da pele, houve um aumento significativo no percentual de pessoas que se autodeclararam como negros. Em relação à distribuição por classes de rendimento per capita, não houve diferenças estatisticamente significativas entre 2013 e 2019. Da mesma forma, a comparação das distribuições por região de residência e situação urbana/rural não mostrou diferenças estatisticamente significativas (Tabela 1).

Quanto às variáveis referentes às condições de saúde, entre 2013 e 2019, foram encontradas diferenças significativas para dois dos três indicadores considerados no estudo: o percentual de pessoas que tiveram problema de saúde nas duas últimas semanas cresceu de 7,0 para $8,1 \%$; a proporção de pessoas que tiveram diagnóstico de alguma doença crônica e não têm limitações para a realização das atividades habituais aumentou de 8,1 a $16,2 \%$, e das que têm doença crônica com limitações, de 6,9 a 15,3\%. Diferenças limítrofes foram encontradas para avaliação do estado de saúde: a proporção de avaliação regular cresceu de 21,6 para $22,3 \%$ e de avaliação ruim de 4,3 para $4,7 \%$ (Tabela 1 ).

Quanto à posse de plano de saúde, não foram encontradas diferenças significativas com proporções variando de 27,9\%, em 2013, 28,5\%, em 2019. Igualmente, os percentuais de pessoas que têm uma fonte usual de cuidados quando precisam de atendimento de saúde não variaram significativamente (77,8\%, em 2013, e 76,5\%, em 2019). Quanto ao tipo de serviço público como fonte usual do cuidado, enquanto para UBS, as proporções permaneceram semelhantes, de 47,8 e $46,8 \%$, em 2013 e 2019 , respectivamente, houve um crescimento nítido de uso de UPA, de 4,1 a $14,1 \%$. No setor privado, houve um aumento significativo para consultório particular, de 20,2 para 22,9\% (Tabela 1).

Na Tabela 2, analisam-se as diferenças nas proporções de pessoas que procuraram atendimento relacionado à saúde nas duas últimas semanas anteriores à pesquisa, segundo os indicadores considerados no estudo. Entre 2013 e 2019, o 
Tabela 1. Distribuição proporcional (\%) da população segundo características demográficas e socioeconômicas, região de residência, indicares de condições de saúde e fonte usual dos cuidados. Brasil, Pesquisa Nacional de Saúde, 2013 e 2019.

\begin{tabular}{|c|c|c|c|c|c|}
\hline \multirow{2}{*}{ Variáveis } & \multirow{2}{*}{ Categoria } & \multicolumn{2}{|c|}{2013} & \multicolumn{2}{|c|}{2019} \\
\hline & & $\%$ & IC 95\% & $\%$ & IC 95\% \\
\hline \multirow[t]{6}{*}{ Faixa de Idade } & $0-4$ & 6,4 & $6,2-6,5$ & 6,1 & $6,0-6,3$ \\
\hline & $5-14$ & 15,4 & $15,1-15,7$ & 13,5 & $13,3-13,8$ \\
\hline & $15-29$ & 24,7 & $24,3-25,0$ & 21,7 & $21,4-22,0$ \\
\hline & $30-44$ & 22,3 & $22,0-22,6$ & 22,6 & $22,3-22,9$ \\
\hline & $45-59$ & 18,1 & $17,8-18,4$ & 19,6 & $19,3-19,9$ \\
\hline & $60+$ & 13,2 & $12,8-13,5$ & 16,4 & $16,0-16,8$ \\
\hline \multirow[t]{2}{*}{ Sexo } & M & 48,1 & $47,9-48,4$ & 47,8 & $47,6-48,0$ \\
\hline & $\mathrm{F}$ & 51,9 & $51,6-52,1$ & 52,2 & $52,0-52,4$ \\
\hline \multirow[t]{4}{*}{ Raça/Cor da Pele } & Branca & 45,9 & $45,2-46,6$ & 43,4 & $42,8-44,1$ \\
\hline & Preta & 8,6 & $8,2-9,0$ & 10,4 & $10,1-10,7$ \\
\hline & Parda & 44,2 & $43,6-44,9$ & 44,9 & $44,3-45,5$ \\
\hline & Outra & 1,2 & $1,1-1,3$ & 1,3 & $1,1-1,4$ \\
\hline \multirow[t]{5}{*}{ Região de residência } & Norte & 8,4 & $8,1-8,6$ & 8,6 & $8,3-9,0$ \\
\hline & Nordeste & 27,6 & $27,1-28,1$ & 27,2 & $26,6-27,8$ \\
\hline & Sudeste & 42,2 & $41,6-42,9$ & 42,2 & $41,3-43,0$ \\
\hline & Sul & 14,3 & $13,9-14,8$ & 14,3 & $13,9-14,7$ \\
\hline & Centro-Oeste & 7,5 & $7,3-7,7$ & 7,7 & $7,4-8,0$ \\
\hline \multirow[t]{2}{*}{ Situação } & Urbana & 85,2 & $84,7-85,7$ & 85,4 & $85,0-85,8$ \\
\hline & Rural & 14,8 & $14,3-15,3$ & 14,6 & $14,2-15,0$ \\
\hline \multirow[t]{5}{*}{ Rendimento per capita } & Até $1 / 2 \mathrm{SM}$ & 26,2 & $25,5-27,0$ & 26,5 & $25,9-27,1$ \\
\hline & $1 / 2$ até $1 \mathrm{SM}$ & 29,9 & $29,1-30,6$ & 29,2 & $28,6-29,8$ \\
\hline & 1 até $2 \mathrm{SM}$ & 25,8 & $25,1-26,4$ & 25,9 & $25,4-26,4$ \\
\hline & 2 até $3 \mathrm{SM}$ & 8,0 & $7,6-8,4$ & 8,2 & $7,9-8,5$ \\
\hline & $3 \mathrm{SM}$ ou mais & 10,1 & $9,5-10,8$ & 10,2 & $9,6-10,7$ \\
\hline Posse de plano de saúde & Sim & 27,9 & $27,1-28,8$ & 28,5 & $27,8-29,2$ \\
\hline \multirow[t]{3}{*}{ Estado de saúde } & Bom & 74,1 & $73,6-74,6$ & 73,0 & $72,6-73,4$ \\
\hline & Regular & 21,6 & $21,1-22,0$ & 22,3 & $22,0-22,7$ \\
\hline & Ruim & 4,3 & $4,2-4,5$ & 4,7 & $4,5-4,8$ \\
\hline $\begin{array}{l}\text { Problema de saúde nas } \\
\text { duas últimas semanas }\end{array}$ & Sim & 7,0 & $6,8-7,2$ & 8,1 & $7,9-8,3$ \\
\hline \multirow{3}{*}{$\begin{array}{l}\text { Tem alguma doença } \\
\text { crônica }\end{array}$} & Não & 85,0 & $84,6-85,4$ & 68,5 & $68,1-68,9$ \\
\hline & Sim, sem limitação & 8,1 & $7,8-8,5$ & 16,2 & $15,9-16,5$ \\
\hline & Sim, com limitações & 6,9 & $6,7-7,1$ & 15,3 & $15,0-15,6$ \\
\hline Fonte usual de cuidados & Sim & 77,8 & $77,0-78,6$ & 76,5 & $75,9-77,1$ \\
\hline \multirow{6}{*}{$\begin{array}{l}\text { Tipo de fonte (entre os } \\
\text { que têm fonte usual de } \\
\text { cuidados) }\end{array}$} & UBS & 47,8 & $46,7-49,0$ & 46,8 & $45,8-47,9$ \\
\hline & UPA & 4,1 & $3,7-4,6$ & 14,1 & $13,4-14,9$ \\
\hline & Outro serviço público & 19,1 & $18,3-20,0$ & 8,9 & $8,5-9,4$ \\
\hline & Consultório particular & 20,2 & $19,4-21,1$ & 22,9 & $22,1-23,6$ \\
\hline & Pronto-atendimento privado & 4,9 & $4,4-5,4$ & 4,4 & $4,0-4,8$ \\
\hline & Outro & 3,9 & $3,6-4,2$ & 2,8 & $2,6-3,1$ \\
\hline
\end{tabular}

percentual de procura por atendimento cresceu de 15,3 a $18,6 \%$; por motivo de doença ou problema de saúde aumentou de 11,5 para $14,7 \%$; para atendimento preventivo, de 1,9 para $3,7 \%$, com o maior aumento relativo para os atendimentos preventivos.

A análise da procura por atendimento relacionado à saúde nas últimas duas semanas 
Tabela 2. Proporção (\%) de pessoas que tiveram atendimento relacionado à saúde nas duas últimas semanas anteriores à pesquisa segundo características demográficas e socioeconômicas, região de residência, indicares de condições de saúde e fonte usual dos cuidados. Brasil, Pesquisa Nacional de Saúde, 2013 e 2019.

\begin{tabular}{|c|c|c|c|c|c|}
\hline \multirow{2}{*}{ Variáveis } & \multirow{2}{*}{ Categoria } & \multicolumn{2}{|c|}{2013} & \multicolumn{2}{|c|}{2019} \\
\hline & & $\%$ & IC 95\% & $\%$ & IC 95\% \\
\hline Total & & 15,3 & $15,0-15,7$ & 18,6 & $18,3-19,0$ \\
\hline \multirow[t]{2}{*}{ Motivo do uso } & Doença & 11,5 & $11,2-11,8$ & 14,7 & $14,4-15,0$ \\
\hline & Prevenção & 1,9 & $1,8-2,0$ & 3,7 & $3,5-3,9$ \\
\hline \multirow[t]{6}{*}{ Faixa de Idade } & $0-4$ & 18,6 & $17,5-19,7$ & 21,7 & $20,6-22,8$ \\
\hline & $5-14$ & 10,1 & $9,4-10,7$ & 12,6 & $12,0-13,3$ \\
\hline & $15-29$ & 10,9 & $10,4-11,4$ & 13,3 & $12,8-13,8$ \\
\hline & $30-44$ & 14,2 & $13,7-14,8$ & 17,2 & $16,6-17,7$ \\
\hline & $45-59$ & 19,0 & $18,2-19,7$ & 22,0 & $21,3-22,6$ \\
\hline & $60+$ & 24,9 & $24,0-25,9$ & 27,5 & $26,8-28,3$ \\
\hline \multirow[t]{2}{*}{ Sexo } & $\mathrm{M}$ & 11,8 & $11,5-12,2$ & 14,8 & $14,5-15,1$ \\
\hline & $\mathrm{F}$ & 18,5 & $18,1-19,0$ & 22,1 & $21,7-22,6$ \\
\hline \multirow[t]{4}{*}{ Raça/Cor da Pele } & Branca & 17,0 & $16,4-17,5$ & 20,2 & $19,7-20,7$ \\
\hline & Preta & 14,7 & $13,7-15,7$ & 19,1 & $18,3-19,9$ \\
\hline & Parda & 13,7 & $13,2-14,1$ & 17,0 & $16,6-17,5$ \\
\hline & Outra & 17,5 & $15,0-20,2$ & 18,9 & $15,6-22,7$ \\
\hline \multirow[t]{5}{*}{ Região de residência } & Norte & 10,0 & $9,5-10,7$ & 13,7 & $13,1-14,3$ \\
\hline & Nordeste & 13,4 & $12,8-13,9$ & 16,6 & $16,1-17,0$ \\
\hline & Sudeste & 17,1 & $16,4-17,7$ & 20,9 & $20,3-21,6$ \\
\hline & $\mathrm{Sul}^{1}$ & 17,9 & $17,0-18,9$ & 19,8 & $19,1-20,5$ \\
\hline & Centro-Oeste & 13,7 & $13,1-14,4$ & 16,7 & $16,0-17,4$ \\
\hline \multirow[t]{2}{*}{ Situação } & Urbana & 15,8 & $15,4-16,2$ & 19,3 & $18,9-19,6$ \\
\hline & Rural & 12,4 & $11,7-13,1$ & 14,8 & $14,3-15,4$ \\
\hline \multirow[t]{5}{*}{ Rendimento per capita } & Até $1 / 2 S M$ & 12,8 & $12,2-13,4$ & 15,3 & $14,8-15,9$ \\
\hline & $1 / 2$ até $1 \mathrm{SM}$ & 14,8 & $14,2-15,3$ & 17,8 & $17,3-18,4$ \\
\hline & 1 até $2 \mathrm{SM}$ & 16,1 & $15,5-16,8$ & 19,4 & $18,7-20,0$ \\
\hline & 2 até $3 \mathrm{SM}$ & 18,0 & $16,8-20,5$ & 21,8 & $20,7-22,9$ \\
\hline & $3 \mathrm{SM}$ ou mais & 19,3 & $18,2-20,5$ & 25,0 & $24,1-25,9$ \\
\hline \multirow[t]{2}{*}{ Posse de plano de saúde } & Não & 13,7 & $13,3-14,1$ & 16,4 & $16,1-16,8$ \\
\hline & Sim & 19,5 & $18,8-20,2$ & 24,1 & $23,5-24,8$ \\
\hline \multirow[t]{3}{*}{ Estado de saúde } & Bom & 11,6 & $11,3-12,0$ & 14,6 & $14,2-14,9$ \\
\hline & Regular & 23,6 & $22,9-24,4$ & 26,8 & $26,1-27,4$ \\
\hline & Ruim & 36,9 & $35,2-38,7$ & 43,2 & $41,7-44,6$ \\
\hline \multirow{2}{*}{$\begin{array}{l}\text { Problema de saúde nas duas } \\
\text { últimas semanas }\end{array}$} & Não & 11,9 & $11,6-12,2$ & 14,8 & $14,5-15,1$ \\
\hline & $\operatorname{Sim}^{1}$ & 61,0 & $59,5-62,6$ & 62,4 & $61,4-63,5$ \\
\hline \multirow[t]{3}{*}{ Tem alguma doença crônica } & Não ${ }^{1}$ & 12,5 & $12,1-12,8$ & 13,0 & $12,7-13,3$ \\
\hline & Sim, sem limitação ${ }^{1}$ & 25,2 & $23,9-26,5$ & 24,5 & $23,7-25,2$ \\
\hline & Sim, com limitações ${ }^{1}$ & 38,6 & $37,2-40,0$ & 37,6 & $36,7-38,4$ \\
\hline \multirow[t]{2}{*}{ Fonte usual do cuidado } & Não & 12,1 & $11,5-12,7$ & 15,8 & $15,2-16,3$ \\
\hline & Sim & 16,2 & $15,8-16,7$ & 19,5 & $19,1-19,9$ \\
\hline
\end{tabular}

${ }^{1}$ Diferenças não significativas no nível de $1 \%$.

por faixa de idade mostra maiores prevalências nos grupos extremos, de 0-4 anos e 60 anos ou mais, tanto em 2013 como em 2019, e o maior crescimento ocorreu entre as crianças menores de 5 anos, de 3,1\%. A comparação das pre- valências por sexo mostra maior procura de atendimento entre as mulheres, mas aumentos significativos para ambos os sexos, entre 2013 e 2019. No tocante à raça/cor da pele, apesar de indivíduos de cor da pele branca apresentarem maior 
prevalência, o crescimento foi mais acentuado para a raça/cor da pele preta, ultrapassando 4 pontos percentuais no período (Tabela 2).

A análise por região de residência aponta para as imensas desigualdades regionais na procura por atendimento de saúde nas duas últimas semanas. Em 2013, as prevalências variaram de $10,0 \%$, na Região Norte, a $17,9 \%$, na Região Sul, e em 2019 , de 13,7\%, na Região Norte, a 20,9\%, na Região Sudeste. Quanto à situação urbana/rural, o maior aumento da prevalência ocorreu entre os residentes de áreas urbanas, intensificando as desigualdades (Tabela 2).

Embora tenham ocorrido aumentos significativos nas prevalências de busca de atendimento de saúde em todas as classes de rendimento per capita no período estudado, as desigualdades por renda permanecem muito pronunciadas. Em 2013, o percentual de pessoas que procuraram atendimento de saúde variou de $12,8 \%$, entre os de pior rendimento per capita, a $19,3 \%$, entre os de maior renda, com uma diferença de 6,5\% entre as classes extremas. Em 2019, a diferença foi ainda mais pronunciada, de $9,7 \%$, com um gradiente de 15,3 a $25,0 \%$, nas classes de pior à de maior rendimento. Entre as pessoas que têm plano de saúde, a prevalência cresceu de 4,6 pontos percentuais, e entre as pessoas que têm uma fonte usual de cuidado, de 3,3 pontos percentuais (Tabela 2).

As maiores proporções de procura de atendimento relacionado à saúde nas duas últimas semanas, tanto em 2013 como em 2019, ocorreram entre as pessoas com necessidade de atenção: superiores a $60 \%$ entre os que tiveram problema de saúde nas duas últimas semanas, sem diferenças significativas entre 2013 e 2019; cerca de $25 \%$ e $38 \%$, entre os que têm uma doença crônica sem limitações e com limitações das atividades habituais, também sem diferenças significativas no período; e aumentando significativamente de 36,9 a 43,2\%, entre 2013 e 2019, entre os que têm avaliação ruim do estado de saúde.

$\mathrm{Na}$ Tabela 3, as proporções de pessoas que consultaram médico nos últimos 12 meses foram analisadas segundo os mesmos indicadores, comparando-se os resultados obtidos nos anos de $2013 \mathrm{e}$ 2019 . O percentual de pessoas que consultaram médico no último ano aumentou de 71,2 para $76,2 \%$. Os maiores percentuais de consulta médica são apresentados por crianças com menos de 5 anos de idade e pelos idosos, alcançando, em 2019, 90,9\% e 86,9\%, respectivamente. As menores prevalências correspondem às faixas de crianças maiores de 5 anos, adolescentes e adultos jovens.
As diferenças por sexo referente ao uso de médico no último ano persistiram, mas diminuíram em 2019, em função do maior aumento para o sexo masculino. Quanto às diferenças por raça/cor da pele, os indivíduos de cor branca detêm prevalências significativamente maiores, embora tenha havido redução na razão de desigualdades entre as prevalências de uso das categorias de raça/cor da pele "branca" e "preta" no período analisado ( $\mathrm{Ta}$ bela 3).

A análise por região de residência indica grandes diferenças na proporção de pessoas que tiveram consulta médica nos últimos 12 meses, variando de $61,4 \%$, na Região Norte, a $75,8 \%$ na Região Sudeste, em 2013, e de 68,0 a $80,6 \%$, em 2019, respectivamente. As prevalências de consulta médica são significativamente maiores entre os residentes de áreas urbanas, com diferenças de cerca de 10 pontos percentuais, em 2013, e de 9 pontos percentuais em 2019 (Tabela 3).

As desigualdades por classes de rendimento per capita são muito pronunciadas, tanto em 2013 como em 2019. As prevalências de consulta médica no último ano variaram de 64,6 a $83,1 \%$, em 2013 , e de 69,7 a $87,6 \%$, em 2019 , alcançando diferenças de 18 pontos percentuais, aproximadamente, entre os extremos inferior e superior das classes de rendimento. Diferenças acentuadas e significativas por posse de plano de saúde foram, igualmente, encontradas nos dois anos analisados: em 2013, o percentual de pessoas que consultaram médico no último ano variou de 65,9 a $84,8 \%$, e em 2019 , de 71,6 a 87,6\%, correspondendo a variações de 19 e 16 pontos percentuais, respectivamente, entre os que têm e os que não têm plano de saúde (Tabela 3).

Considerando os indicadores de condições de saúde, as proporções de indivíduos que consultaram médico no último ano crescem à medida que aumenta a necessidade. De acordo com o estado de saúde, as proporções variaram de 67,7 a $87,8 \%$, entre as pessoas que têm avaliação boa e ruim, em 2013, e de 73,0 a $91,8 \%$, em 2019 , respectivamente. Considerando os indivíduos que tiveram problemas de saúde nas duas últimas semanas, as prevalências alcançaram mais de $92 \%$, tanto em 2013 como em 2019. Grandes prevalências de consulta médica no último ano são encontradas entre as pessoas com diagnóstico de alguma doença crônica, sem e com limitações das atividades habituais, de cerca de $88 \mathrm{e}$ $92 \%$, respectivamente (Tabela 3 ).

Na Tabela 4, são apresentados os resultados dos modelos multivariados de regressão de Poisson para os indivíduos com 30 anos ou mais de idade. 
Tabela 3. Proporção (\%) de pessoas que consultaram médico nos últimos 12 meses anteriores à pesquisa segundo características demográficas e socioeconômicos, região de residência, indicares de condições de saúde e fonte usual dos cuidados. Brasil, Pesquisa Nacional de Saúde, 2013 e 2019.

\begin{tabular}{|c|c|c|c|c|c|}
\hline \multirow{2}{*}{ Variáveis } & \multirow{2}{*}{ Categoria } & \multicolumn{2}{|c|}{2013} & \multicolumn{2}{|c|}{2019} \\
\hline & & $\%$ & IC 95\% & $\%$ & IC 95\% \\
\hline Total & & 71,2 & $70,7-71,7$ & 76,2 & $75,8-76,5$ \\
\hline \multirow[t]{6}{*}{ Faixa de Idade } & $0-4$ & 87,7 & $86,8-88,6$ & 90,9 & $90,2-91,6$ \\
\hline & $5-14$ & 66,5 & $65,5-67,5$ & 72,7 & $71,7-73,6$ \\
\hline & $15-29$ & 62,7 & $61,9-63,6$ & 67,0 & $66,3-67,7$ \\
\hline & $30-44$ & 69,0 & $68,2-69,8$ & 73,1 & $72,5-73,7$ \\
\hline & $45-59$ & 74,6 & $73,7-75,4$ & 78,6 & $78,0-79,2$ \\
\hline & $60+$ & 83,5 & $82,8-84,2$ & 86,9 & $86,4-87,4$ \\
\hline \multirow[t]{2}{*}{ Sexo } & M & 63,9 & $63,2-64,6$ & 69,4 & $69,0-69,9$ \\
\hline & $\mathrm{F}$ & 78,0 & $77,5-78,5$ & 82,3 & $82,0-82,7$ \\
\hline \multirow[t]{4}{*}{ Raça/Cor da Pele } & Branca & 74,8 & $74,1-75,5$ & 79,4 & $78,9-79,9$ \\
\hline & Preta & 69,5 & $68,1-70,9$ & 74,8 & $73,9-75,7$ \\
\hline & Parda & 67,7 & $67,1-68,4$ & 73,3 & $72,8-73,8$ \\
\hline & Outra & 73,9 & $71,0-76,6$ & 78,7 & $75,0-82,0$ \\
\hline \multirow[t]{5}{*}{ Região de residência } & Norte & 61,4 & $59,9-62,8$ & 68,0 & $66,9-69,0$ \\
\hline & Nordeste & 66,3 & $65,5-67,1$ & 71,9 & $71,3-72,5$ \\
\hline & Sudeste & 75,8 & $74,8-76,8$ & 80,6 & $79,9-81,2$ \\
\hline & Sul & 73,8 & $72,6-74,9$ & 77,7 & $76,9-78,5$ \\
\hline & Centro-Oeste & 69,5 & $68,4-70,6$ & 73,7 & $72,6-74,8$ \\
\hline \multirow[t]{2}{*}{ Situação } & Urbana & 72,7 & $72,1-73,2$ & 77,5 & $77,1-77,9$ \\
\hline & Rural & 62,8 & $61,5-64,0$ & 68,6 & $67,8-69,4$ \\
\hline \multirow[t]{5}{*}{ Rendimento per capita } & Até $1 / 2 \mathrm{SM}$ & 64,6 & $63,6-65,5$ & 69,7 & $69,0-70,5$ \\
\hline & $1 / 2$ até $1 \mathrm{SM}$ & 69,3 & $68,4-70,1$ & 74,6 & $74,0-75,2$ \\
\hline & 1 até 2 SM & 73,7 & $72,7-74,6$ & 78,1 & $77,5-78,7$ \\
\hline & 2 até $3 \mathrm{SM}$ & 77,1 & $75,6-78,5$ & 82,4 & $81,4-83,5$ \\
\hline & $3 \mathrm{SM}$ ou mais & 83,1 & $82,1-84,1$ & 87,6 & $86,8-88,4$ \\
\hline \multirow[t]{2}{*}{ Posse de plano de saúde } & Não & 65,9 & $65,3-66,6$ & 71,6 & $71,2-72,1$ \\
\hline & Sim & 84,8 & $84,0-85,5$ & 87,6 & $87,1-88,1$ \\
\hline \multirow[t]{3}{*}{ Estado de saúde } & Bom & 67,7 & $67,1-68,4$ & 73,0 & $72,5-73,4$ \\
\hline & Regular & 79,9 & $79,1-80,6$ & 83,3 & $82,8-83,8$ \\
\hline & Ruim & 87,8 & $86,5-88,9$ & 91,8 & $91,0-92,5$ \\
\hline \multirow{2}{*}{$\begin{array}{l}\text { Problema de saúde nas duas } \\
\text { últimas semanas }\end{array}$} & Não & 69,6 & $69,0-70,1$ & 74,6 & $74,2-75,0$ \\
\hline & $\operatorname{Sim}^{1}$ & 92,8 & $92,0-93,5$ & 94,0 & $93,5-94,5$ \\
\hline \multirow[t]{3}{*}{ Tem alguma doença crônica } & Não & 67,8 & $67,2-68,4$ & 69,7 & $69,2-70,2$ \\
\hline & Sim, sem limitação ${ }^{1}$ & 88,8 & $87,9-89,7$ & 88,0 & $87,5-88,6$ \\
\hline & Sim, com limitações ${ }^{1}$ & 92,4 & $91,6-93,1$ & 92,6 & $92,2-93,0$ \\
\hline \multirow[t]{2}{*}{ Fonte usual do cuidado } & Não & 63,2 & $62,2-64,3$ & 69,7 & $68,9-70,5$ \\
\hline & Sim & 73,5 & $72,9-74,1$ & 78,2 & $77,8-78,6$ \\
\hline
\end{tabular}

${ }^{1}$ Diferenças não significativas no nível de $1 \%$.

Considerando como variável resposta "Procura de atendimento por motivo de doença ou problema de saúde", tanto em 2013 como em 2019, foram encontradas: maiores prevalências de procura com o aumento da idade; razões de prevalências 50\% maiores entre as mulheres em comparação com os homens; $12 \%$ maiores entre os moradores de áre- as urbanas comparados aos de áreas rurais; e cerca de $25 \%$ maiores entre os que têm uma fonte usual de cuidados. As diferenças entre as regiões Sudeste e Sul e a Norte são significativas e acentuadas nos dois anos considerados. Já a associação da variável de rendimento per capita com o desfecho é estatisticamente não significativa, após o controle de ida- 
Tabela 4. Fatores associados (considerando rendimento per capita como covariável) com a procura de atendimento relacionado à saúde nas duas últimas semanas anteriores à pesquisa de acordo com o motivo (problema de saúde ou prevenção) entre indivíduos de 30 anos ou mais. Brasil, Pesquisa Nacional de Saúde, 2013 e 2019.

\begin{tabular}{|c|c|c|c|c|c|}
\hline \multicolumn{6}{|c|}{ Procura de atendimento por problema de saúde } \\
\hline \multirow{2}{*}{ Variáveis } & \multirow{2}{*}{ Categoria } & \multicolumn{2}{|c|}{2013} & \multicolumn{2}{|c|}{2019} \\
\hline & & RP ajustada & Valor de p & RP ajustada & Valor de $\mathrm{p}$ \\
\hline Idade & & 1,02 & $<0,001$ & 1,01 & $<0,001$ \\
\hline \multirow[t]{2}{*}{ Sexo } & M & 1,00 & - & 1,00 & - \\
\hline & $\mathrm{F}$ & 1,50 & $<0,001$ & 1,46 & $<0,001$ \\
\hline \multirow[t]{5}{*}{ Região de residência } & Norte & 1,00 & - & 1,00 & - \\
\hline & Nordeste & 1,04 & 0,420 & 1,13 & $<0,001$ \\
\hline & Sudeste & 1,37 & $<0,001$ & 1,31 & $<0,001$ \\
\hline & Sul & 1,53 & $<0,001$ & 1,28 & $<0,001$ \\
\hline & Centro-Oeste & 1,09 & 0,085 & 1,15 & $<0,001$ \\
\hline \multirow[t]{2}{*}{ Situação } & Urbana & 1,12 & 0,002 & 1,12 & $<0,001$ \\
\hline & Rural & 1,00 & - & 1,00 & - \\
\hline \multirow[t]{2}{*}{ Fonte usual de cuidados } & Não & 1,00 & - & 1,00 & - \\
\hline & Sim & 1,28 & $<0,001$ & 1,25 & $<0,001$ \\
\hline \multicolumn{2}{|c|}{ Rendimento per capita (logaritmo) } & 0,98 & 0,081 & 1,00 & 0,608 \\
\hline \multicolumn{6}{|c|}{ Procura de atendimento para prevenção } \\
\hline \multirow{2}{*}{ Variáveis } & \multirow{2}{*}{ Categoria } & \multicolumn{2}{|c|}{2013} & \multicolumn{2}{|c|}{2019} \\
\hline & & RP ajustada & Valor de p & RP ajustada & Valor de $\mathrm{p}$ \\
\hline Idade & & 1,00 & 0,866 & 1,01 & 0,001 \\
\hline \multirow[t]{2}{*}{ Sexo } & M & 1,00 & - & 1,00 & - \\
\hline & $\mathrm{F}$ & 2,43 & $<0,001$ & 1,68 & $<0,001$ \\
\hline \multirow[t]{5}{*}{ Região de residência } & Norte & 1,00 & - & 1,00 & - \\
\hline & Nordeste & 1,94 & $<0,001$ & 1,22 & 0,004 \\
\hline & Sudeste & 1,73 & $<0,001$ & 1,23 & 0,007 \\
\hline & Sul & 1,68 & 0,001 & 1,14 & 0,101 \\
\hline & Centro-Oeste & 1,45 & 0,013 & 1,01 & 0,861 \\
\hline \multirow[t]{2}{*}{ Situação } & Urbana & 1,23 & 0,088 & 1,46 & $<0,001$ \\
\hline & Rural & 1,00 & - & 1,00 & - \\
\hline \multirow[t]{2}{*}{ Fonte usual de cuidados } & Não & 1,00 & - & 1,00 & - \\
\hline & Sim & 1,18 & 0,083 & 1,07 & 0,214 \\
\hline \multicolumn{2}{|c|}{ Rendimento per capita (logaritmo) } & 1,21 & $<0,001$ & 1,29 & $<0,001$ \\
\hline
\end{tabular}

de, sexo, situação urbana/rural, região de residência e ter uma fonte usual do cuidado, tanto em 2013 como em 2019.

Ainda na Tabela 4, a comparação dos dois desfechos, "procura de atendimento relacionado à saúde por problema de saúde" com "procura de atendimento preventivo" mostra maiores razões de prevalências para sexo e situação urbana/rural, e com diferenças significativas entre as regiões Nordeste e Sudeste em relação à Norte. Quanto a ter uma fonte usual de cuidado, as razões de prevalência passam a ser não significativas, enquanto as associações com rendimento per capita são significativas no nível de $1 \%$ (RP ajustada $=1,21$, em 2013, e $\mathrm{RP}$ ajustada $=1,29$, em 2019).

$\mathrm{Na}$ Tabela 5, foram apresentados os resultados dos modelos multivariados, substituindo-se a variável de rendimento per capita por posse de plano. Em relação à procura de atendimento por motivo de doença ou problema de saúde, as associações com idade, sexo, situação urbana/rural, ter uma fonte usual de cuidados, e as diferenças por região foram semelhantes às encontradas nos modelos apresentados na Tabela 4. Contudo, entre as pessoas que têm plano de saúde, as prevalências de procura 
Tabela 5. Fatores associados (considerando posse de plano de saúde como covariável) com a procura de atendimento relacionado à saúde nas duas últimas semanas anteriores à pesquisa de acordo com o motivo (problema de saúde ou prevenção) entre indivíduos de 30 anos ou mais. Brasil, Pesquisa Nacional de Saúde, 2013 e 2019.

\begin{tabular}{|c|c|c|c|c|c|}
\hline \multicolumn{6}{|c|}{ Procura de atendimento por problema de saúde } \\
\hline \multirow{2}{*}{ Variáveis } & \multirow{2}{*}{ Categoria } & \multicolumn{2}{|c|}{2013} & \multicolumn{2}{|c|}{2019} \\
\hline & & RP ajustada & Valor de p & RP ajustada & Valor de p \\
\hline Idade & & 1,02 & $<0,001$ & 1,01 & $<0,001$ \\
\hline \multirow[t]{2}{*}{ Sexo } & M & 1,00 & - & 1,00 & - \\
\hline & $\mathrm{F}$ & 1,50 & $<0,001$ & 1,45 & $<0,001$ \\
\hline \multirow[t]{5}{*}{ Região de residência } & Norte & 1,00 & - & 1,00 & - \\
\hline & Nordeste & 1,04 & 0,447 & 1,13 & $<0,001$ \\
\hline & Sudeste & 1,33 & $<0,001$ & 1,28 & $<0,001$ \\
\hline & Sul & 1,48 & $<0,001$ & 1,25 & $<0,001$ \\
\hline & Centro-Oeste & 1,07 & 0,212 & 1,13 & 0,001 \\
\hline \multirow[t]{2}{*}{ Situação } & Urbana & 1,09 & 0,031 & 1,00 & - \\
\hline & Rural & 1,00 & - & 1,08 & 0,002 \\
\hline \multirow[t]{2}{*}{ Fonte usual de cuidados } & Não & 1,00 & - & 1,00 & - \\
\hline & Sim & 1,27 & $<0,001$ & 1,24 & $<0,001$ \\
\hline \multirow[t]{2}{*}{ Posse de plano de saúde } & Não & 1,00 & - & 1,00 & - \\
\hline & Sim & 1,11 & 0,001 & 1,16 & $<0,001$ \\
\hline \multicolumn{6}{|c|}{ Procura de atendimento para prevenção } \\
\hline \multirow{2}{*}{ Variáveis } & \multirow{2}{*}{ Categoria } & \multicolumn{2}{|c|}{2013} & \multicolumn{2}{|c|}{2019} \\
\hline & & RP ajustada & Valor de p & RP ajustada & Valor de $p$ \\
\hline Idade & & 1,00 & 0,675 & 1,01 & $<0,001$ \\
\hline \multirow[t]{2}{*}{ Sexo } & M & 1,00 & - & 1,00 & - \\
\hline & $\mathrm{F}$ & 2,37 & $<0,001$ & 1,62 & $<0,001$ \\
\hline \multirow[t]{5}{*}{ Região de residência } & Norte & 1,00 & - & 1,00 & - \\
\hline & Nordeste & 1,90 & $<0,001$ & 1,20 & 0,007 \\
\hline & Sudeste & 1,67 & $<0,001$ & 1,20 & 0,015 \\
\hline & Sul & 1,68 & 0,001 & 1,17 & 0,056 \\
\hline & Centro-Oeste & 1,43 & 0,015 & 1,04 & 0,645 \\
\hline \multirow[t]{2}{*}{ Situação } & Urbana & 1,18 & 0,164 & 1,37 & $<0,001$ \\
\hline & Rural & 1,00 & - & 1,00 & - \\
\hline \multirow[t]{2}{*}{ Fonte usual de cuidados } & Não & 1,00 & - & 1,00 & - \\
\hline & Sim & 1,15 & 0,145 & 1,04 & 0,487 \\
\hline \multirow[t]{2}{*}{ Posse de plano de saúde } & Não & 1,00 & - & 1,00 & - \\
\hline & Sim & 1,77 & $<0,001$ & 2,10 & $<0,001$ \\
\hline
\end{tabular}

de atendimento foram significativamente maiores, com razões de prevalências ajustadas de 1,11 e 1,16 em 2013 e 2019, respectivamente. No que se refere à procura de atendimento preventivo, as razões de prevalências ajustadas são bem maiores, de 1,77, em 2013, e alcançando 2,10 em 2019, ou seja, os indivíduos que têm plano de saúde têm prevalência duas vezes maior de procura de atendimento para prevenção.

\section{Discussão}

Os resultados do presente estudo mostram crescimento tanto na procura como na utilização dos serviços de saúde, com aumentos na proporção de indivíduos que procuraram atendimento relacionado à saúde nas duas semanas anteriores à pesquisa e na proporção de pessoas que consultaram médico nos últimos 12 meses anteriores à pesquisa, quando os dados da PNS 2019 são 
comparados aos da PNS 2013. Os benefícios da ampliação da cobertura da utilização de serviços de saúde são devidamente reconhecidos, uma vez que a expansão da prevenção, diagnóstico e tratamento precoce de doenças podem resultar na redução da mortalidade prematura, no aumento da expectativa de vida com qualidade e na diminuição das hospitalizações desnecessárias ${ }^{18}$.

Entretanto, a interpretação do aumento na utilização dos serviços de saúde não é imediata, uma vez que ela depende de vários fatores, como a necessidade, as características sociodemográficas, a oferta de serviços, a disponibilidade de médicos, o financiamento da assistência de saúde, e a percepção dos usuários em relação à assistência prestada $^{19}$.

Os achados deste trabalho mostram o envelhecimento da população brasileira entre 2013 e 2019, junto ao crescimento das prevalências de doenças crônicas, gerando mais necessidades de atenção em saúde, limitações nas atividades da vida diária e maiores dificuldades de acesso aos serviços de saúde entre os idosos ${ }^{20}$. Desafios relacionados a minimizar as barreiras de acesso entre os idosos, a ofertar mais serviços, e a prover financiamento da prevenção e do tratamento das doenças crônicas não transmissíveis têm sido enfrentados, igualmente, em outros países ${ }^{21-23}$.

É interessante notar que tocante à raça/cor da pele, houve um aumento significativo no percentual de pessoas que se autodeclararam como negros, entre 2013 e 2019, corroborando resultados de análise de coortes de nascimento com os dados da Pesquisa Nacional de Amostra de Domicílios, que mostram que as coortes tornamse menos "brancas" a cada ano, principalmente após os anos 2000. As variações nas distribuições proporcionais por raça/cor da pele são atribuídas a mudanças na autoidentificação da cor da pele durante a vida, em um movimento consistente de maior percentual de declaração da cor da pele preta em detrimento da branca ${ }^{24}$.

Em relação às diferenças de utilização de serviços de saúde por sexo, maiores percentuais de procura de atendimento e de utilização de médico foram encontrados entre as mulheres. Em geral, estudos que abordam a morbidade e a utilização de serviços de saúde relatam que as mulheres têm pior percepção do estado de saúde e são mais propensas a usar cuidados à saúde ${ }^{13}$. Quanto à idade, as maiores frequências de busca de atendimento relacionado à saúde ocorrem entre os com mais necessidade de atenção, as crianças com menos de 5 anos e os idosos, corroborando achados de estudos anteriores ${ }^{25,26}$.
Ter um problema de saúde continuou sendo o principal motivo de busca por serviços, em 2013 e 2019. Contudo, houve um maior aumento relativo na proporção de atendimentos para prevenção, sugerindo que o envelhecimento da população brasileira e o aumento da necessidade de assistência de saúde são possíveis explicações para a maior utilização de serviços de saúde, mas outros fatores podem ter influenciado a ampliação da busca de atendimento. A Política Nacional de Promoção da Saúde (PNPS) foi implementada em meados dos anos 2000 e proveu maior disponibilidade de serviços de prevenção na atenção primária em saúde. Estudo realizado em 2014 mostrou que programas de promoção da saúde estavam presentes na maioria das unidades básicas de saúde ${ }^{27}$.

Em relação à posse de plano de saúde, não houve aumento significativo entre 2013 e 2019, com mais de $70 \%$ da população fazendo uso do sistema público ${ }^{28}$. Ter uma fonte usual do cuidado foi um fator preponderante para a busca de atendimento de saúde. A principal fonte de cuidados no SUS foi a rede básica, com cerca de $60 \%$ dos atendimentos em estabelecimentos públicos realizados nas UBS, nos dois anos considerados, conforme já apontado em publicação com dados da PNS 201329. Todavia, de 2013 a 2019, houve um crescimento significativo no uso das UPA como fonte usual cuidado, mostrando que a expansão do acesso foi relevante para a obtenção da assistência, mas nem sempre é adequada se não responder, inteiramente, às necessidades do usuário $^{30}$. Estudo realizado nos Estados Unidos mostra que a prestação de cuidados primários através de serviços alternativos pode superar algumas barreiras de acesso, porém a falta de integração na rede de serviços pode implicar na insatisfação dos usuários sobre a qualidade do serviço pres$\operatorname{tado}^{31}$.

A proporção de indivíduos que consultou médico no último ano aumentou consideravelmente, em 5 pontos percentuais, alcançando $76 \%$, em 2019, em patamar semelhante ao de alguns países desenvolvidos ${ }^{32}$. Entre os indivíduos que tinham planos de saúde, a prevalência foi maior, alcançando 88\%, em 2019, mas o aumento na utilização de médico $(5,7$ pontos percentuais) foi maior entre os usuários do SUS do que entre os que têm plano de saúde (2,8 pontos percentuais).

A posse de plano de saúde pode mitigar a existência de possíveis barreiras financeiras no momento do consumo de serviços, como também pode levar a uma resposta mais oportuna 
com a redução do tempo de espera para o atendimento e à maior satisfação do usuário ${ }^{19}$. Conforme discutido em outros artigos nacionais, a posse de plano/seguro de saúde não só aumenta a realização de consultas médicas ${ }^{14,28}$, como também é um determinante da maior utilização de serviços preventivos $^{33}$.

A análise dos modelos multivariados, tanto em 2013 como em 2019, evidenciou que na situação de procura de atendimento por doença ou problema de saúde, não houve associação significativa com nível de renda, após o controle de idade, sexo, regiões de residência, situação urbana/rural, e ter uma fonte usual de cuidado. Esses resultados indicam que os serviços de saúde são acessíveis para os indivíduos com percepção dos seus problemas de saúde, independentemente do nível de renda, e são particularmente relevantes no contexto da redução das desigualdades socioeconômicas em saúde.

Entretanto, a análise multivariada dos dados das duas edições da PNS relativos à busca de atendimento por região de residência, mostra que as desigualdades regionais são ainda pronunciadas, mesmo controlando os efeitos de rendimento e de posse de pano de saúde, e independentemente do motivo de procura. As desigualdades regionais no uso de serviços de saúde são apontadas desde 1998, com maiores prevalências nas regiões Sudeste e Sul, e a menor na região Norte ${ }^{34}$. $\mathrm{O}$ acesso diferenciado aos serviços prestados e a desigualdade na distribuição geográfica dos recursos disponíveis têm sido considerados como os principais mecanismos explicativos ${ }^{35,36}$.

Os resultados deste trabalho mostram o bom desempenho do sistema nacional de saúde no que se refere à expansão da cobertura de serviços de saúde, permitindo atender às mudanças nas ne- cessidades de saúde da população, apesar do subfinanciamento crônico do SUS e das políticas de austeridade após o ano de 2016. As desigualdades regionais indicam, entretanto, que as necessidades de saúde da população residente nas regiões menos desenvolvidas não estão sendo atendidas e devem ser objeto de estudos complementares para subsidiar o planejamento de estratégias com vistas à superação das desigualdades. Diante do envelhecimento da população brasileira, do aumento da obesidade e da prevalência de doenças crônicas, e a premência de adoção dos comportamentos saudáveis pela população brasileira, modelos de atenção mais focados na prevenção e promoção da saúde são necessários ${ }^{37}$.

Uma das limitações deste trabalho se refere às diferenças nos questionários das duas pesquisas. Em 2019, houve a exclusão do módulo sobre a avaliação do atendimento médico, não sendo possível saber o motivo da consulta médica no último ano (problema de saúde ou prevenção) e impossibilitando a realização da análise multivariada similar à apresentada para o desfecho "Procura de atendimento de saúde nos 15 dias anteriores à pesquisa”.

Outra limitação é que os indicadores de utilização de serviços de saúde são baseados em dados referidos, que, reconhecidamente, são sujeitos ao viés de memória. Adicionalmente, as perguntas desse módulo da PNS podem ser respondidas pelo informante-chave do domicílio, aumentando a possibilidade de erros de medição de eventos passados. Finalmente, devido ao grande tamanho de amostra nas duas edições da PNS, pequenas diferenças podem ser consideradas estatisticamente significativas e as associações encontradas neste trabalho devem ser examinadas à luz desta limitação. 


\section{Colaboradores}

CL Szwarcwald - participou na concepção e delineamento, análise e interpretação dos dados, redação do artigo. SR Stopa, GN Damacena, WS Almeida, PRB Souza Júnior, MLFP Vieira, CA Pereira - participaram da análise e interpretação dos dados, redação e revisão crítica do artigo. LMV Sardinha, EM Macário - participaram da concepção e delineamento, redação e revisão crítica do artigo. Todos os autores aprovaram a versão final do manuscrito.

\section{Referências}

1. Paim J, Travassos C, Almeida C, Bahia L, Macinko J. The Brazilian health system: history, advances, and challenges. Lancet 2011; 377(9779):1778-1797.

2. Escorel S, Giovanella L, Mendonça MHM, Senna MCM. The Family Health Program and the construction of a new model for primary care in Brazil. Rev Panam Salud Publica 2007; 21(2-3):164-176.

3. Giovanella L, Mendonça MH, de Almeida PF, Escorel S, Senna MC, Fausto MC, Delgado MM, Andrade CL, Cunha MS, Martins MI, Teixeira CP. Family health: limits and possibilities for an integral primary care approach to health care in Brazil. Cien Saude Colet 2009; 14(3):783-794.

4. Castro MC, Massuda A, Almeida G, Menezes-Filho NA, Andrade MV, Souza Noronha KVM, Rocha R, Macinko J, Hone T, Tasca R, Giovanella L, Malik AM, Werneck H, Fachini LA, Atun R. Brazil's unified health system: the first 30 years and prospects for the future. Lancet 2019; 394(10195):345-356.

5. Lima LD, Albuquerque MV, Scatena JHG, Melo ECP, Oliveira EXG, Carvalho MS, Pereira AMM, Oliveira RAD, Martinelli NL, Oliveira CF. Regional governance arrangements of the Brazilian Unified National Health System: provider diversity and spacial inequality in service provision. Cad Saude Publica 2019; 35(Supl. 2):e00094618.

6. Peltzer K. Patient experiences and health system responsiveness in South Africa. BMC Health Serv Res 2009; 9:117.

7. Birch S, Gafni A. Achievements and challenges of medicare in Canada: Are we there yet? Are we on course? Int J Health Serv 2005; 35(3):443-463.

8. Macinko J, Guanais FC. Population experiences of primary care in 11 Organization for Economic Cooperation and Development countries. Int J Qual Health Care 2015; 27(6):443-450.

9. Gouveia GC, Souza WV, Luna CF, Souza-Júnior PR, Szwarcwald CL. Health care users' satisfaction in Brazil, 2003. Cad Saude Publica 2005; 21(Supl. 1):109118.

10. Travassos C, Viacava F. Utilização e financiamento de serviços de saúde: dez anos de informação das PNAD. Cien Saude Colet 2011; 16(9):3646-3647.

11. Szwarcwald CL, Malta DC, Pereira CA, Vieira ML, Conde WL, Souza Júnior PR, Damacena GN, Azevedo LO, Azevedo E, Silva G, Theme Filha MM, Lopes CS, Romero DE, Almeida WS, Monteiro CA. Pesquisa Nacional de Saúde no Brasil: concepção e metodologia de aplicação. Cien Saude Colet 2014; 19(2):333-342.

12. Malta DC, Silva MMAD, Moura L, Morais Neto OL. The implantation of the Surveillance System for Non-communicable Diseases in Brazil, 2003 to 2015: successes and challenges. Rev Bras Epidemiol 2017; 20(4):661-675.

13. Silva ZP, Ribeiro MC, Barata RB, Almeida MF. Perfil sociodemográfico e padrão de utilização dos serviços de saúde do Sistema Único de Saúde (SUS), 20032008. Cien Saude Colet 2011; 16(9):3807-3816.

14. Pilotto LM, Celeste RK. Tendências no uso de serviços de saúde médicos e odontológicos e a relação com nível educacional e posse de plano privado de saúde no Brasil, 1998-2013. Cad Saude Publica 2018; 34(4): e00052017. 
15. Souza-Jr PRB, Freitas MPS, Antonaci GA, Szwarcwald CL. Desenho da amostra da Pesquisa Nacional de Saúde 2013. Epidemiol Serv Saúde 2015; 24(2):207216.

16. Instituto Brasileiro de Geografia e Estatística (IBGE). Nota Técnica - Informações referentes à revisão do plano tabular da PNS 2013. [nota técnica internet]. 2020 Set [acessado 2020 Nov 03]; [2p.]. Disponível em: https://ftp.ibge.gov.br/PNS/Documentacao_Geral/ Nota_Tecnica_Revisao_Plano_Tabular_PNS2013.pdf

17. Stopa SR, Szwarcwald CL, Oliveira MM, Gouvea ECDP, Vieira MLFP, Freitas MPS, Sardinha LMV, Macário EM. National Health Survey 2019: history, methods and perspectives. Epidemiol Serv Saude 2020; 29(5):e2020315.

18. Macinko J, Dourado I, Aquino R, Bonolo PdeF, Lima-Costa MF, Medina MG, Mota E, Oliveira VB, Turci MA. Major expansion of primary care in Brazil linked to decline in unnecessary hospitalization. Health Aff (Millwood) 2010; 29(12):2149-2160.

19. Szwarcwald CL, Damacena GN, Souza Júnior PR, Almeida WS, Malta DC. Perception of the Brazilian population on medical health care. Brazil, 2013. Cien Saude Colet 2016; 21(2):339-349.

20. Louvison MC, Lebrão ML, Duarte YA, Santos JL, Malik AM, Almeida ES. Desigualdades no uso e acesso aos serviços de saúde entre idosos do município de São Paulo. Rev Saude Publica 2008; 42(4):733-740.

21. Zhang T, Liu J, Liu C. Changes in Perceived Accessibility to Healthcare from the Elderly between 2005 and 2014 in China: An Oaxaca-Blinder Decomposition Analysis. Int J Environ Res Public Health 2019; 16(20):3824.

22. Barr ML, Welberry H, Comino EJ, Harris-Roxas BF, Harris E, Lloyd J, Whitney S, O'Connor C, Hall J, Harris MF. Understanding the use and impact of allied health services for people with chronic health conditions in Central and Eastern Sydney, Australia: a fiveyear longitudinal analysis. Prim Health Care Res Dev 2019; 20:e141.

23. Jayathilaka R, Joachim S, Mallikarachchi V, Perera N, Ranawaka D. Chronic diseases: An added burden to income and expenses of chronically-ill people in Sri Lanka. PLoS One 2020; 15(10):e0239576.

24. Soares AS. A demografia da cor: a composição brasileira de 1890 a 2007. In: Theodoro M, organizador. As políticas púbicas e a desigualdade racial no Brasil: 120 anos após a abolição. Brasília: IPEA; 2008.

25. Oliveira BLCA, Moreira JPL, Luiz RR. The influence of the Family Healthcare Strategy in the use of healthcare services by children in Brazil: an analysis using the Propensity Score Matching (PSM) method of National Health Survey data. Cien Saude Colet 2019; 24(4):1495-1505.

26. Malta DC, Bernal RTI, Lima MG, Araújo SSC, Silva MMAD, Freitas MIF, Barros MBA. Noncommunicable diseases and the use of health services: analysis of the National Health Survey in Brazil. Rev Saude Publica 2017; 51(Supl. 1):4s.

27. Ramos LR, Malta DC, Gomes GA, Bracco MM, Florindo AA, Mielke GI, Parra DC, Lobelo F, Simoes EJ, Hallal PC. Prevalence of health promotion programs in primary health care units in Brazil. Rev Saude Publica 2014; 48(5):837-844.
28. Stopa SR, Malta DC, Monteiro CN, Szwarcwald CL, Goldbaum M, Cesar CLG. Use of and access to health services in Brazil, 2013 National Health Survey. Rev Saude Publica 2017; 51(Supl. 1):3s.

29. Dourado I, Medina MG, Aquino R. The effect of the Family Health Strategy on usual source of care in Brazil: data from the 2013 National Health Survey (PNS 2013). Int J Equity Health 2016; 15(1):151.

30. Lima-Costa MF, Turci MA, Macinko J. A comparison of the Family Health Strategy to other sources of healthcare: utilization and quality of health services in Belo Horizonte, Minas Gerais State, Brazil. Cad Saude Publica 2013; 29(7):1370-1380.

31. Reed C, Rabito FA, Werthmann D, Smith S, Carlson JC. Factors associated with using alternative sources of primary care: a cross-sectional study. BMC Health Serv Res 2019; 19(1):933.

32. Van Doorslaer E, Masseria C, Koolman X; for the OECD Health Equity Research Group. Inequalities in access to medical care by income in developed countries. CMAJ 2006; 174(2):177-183.

33. Malta DC, Bernal RTI, Vieira Neto E, Curci KA, Pasinato MTM, Lisbôa RM, Cachapuz RF, Coelho KSC, Santos FPD, Freitas MIF. Noncommunicable diseases, risk factors, and protective factors in adults with and without health Insurance. Cien Saude Colet 2020; 25(8):2973-2983.

34. Viacava F, Porto SM, Carvalho CC, Bellido JG. Health inequalities by region and social group based on data from household surveys (Brazil, 1998-2013). Cien Saude Colet 2019; 24(7):2745-2760.

35. Oliveira RAD, Duarte CMR, Pavão ALB, Viacava F. Barriers in access to services in five Health Regions of Brazil: perceptions of policymakers and professionals in the Brazilian Unified National Health System. Cad Saude Publica 2019; 35(11):e00120718.

36. Nogueira MC, Fayer VA, Corrêa CSL, Guerra MR, Stavola B, Dos-Santos-Silva I, Bustamante-Teixeira MT, Silva GAE. Inequities in access to mammographic screening in Brazil. Cad Saude Publica 2019; 35(6):e00099817.

37. Paim JS. Thirty years of the Unified Health System (SUS). Cien Saude Colet 2018; 23(6):1723-1728.

Artigo apresentado em 11/11/2020

Aprovado em 01/12/2020

Versão final apresentada em 03/12/2020

Editores-chefes: Maria Cecília de Souza Minayo, Romeu Gomes, Antônio Augusto Moura da Silva 